\title{
Artificial fingerprint recognition by using optical coherence tomography with autocorrelation analysis
}

\author{
Yezeng Cheng and Kirill V. Larin
}

\begin{abstract}
Fingerprint recognition is one of the most widely used methods of biometrics. This method relies on the surface topography of a finger and, thus, is potentially vulnerable for spoofing by artificial dummies with embedded fingerprints. In this study, we applied the optical coherence tomography (OCT) technique to distinguish artificial materials commonly used for spoofing fingerprint scanning systems from the real skin. Several artificial fingerprint dummies made from household cement and liquid silicone rubber were prepared and tested using a commercial fingerprint reader and an OCT system. While the artificial fingerprints easily spoofed the commercial fingerprint reader, OCT images revealed the presence of them at all times. We also demonstrated that an autocorrelation analysis of the OCT images could be potentially used in automatic recognition systems. (C) 2006 Optical Society of America
\end{abstract}

OCIS codes: $\quad 100.5010,110.4500,160.4670,170.6930$.

\section{Introduction}

Accurate automatic identification and recognition of a person is currently considered a cornerstone of many security applications, especially in this modern digital age. There are several different biometric techniques to recognize a person. Behavioral characteristics, such as keystrokes dynamics and signature dynamics, and physical characteristics, such as iris recognition, face recognition, and fingerprint recognition, are becoming the dominant methods for biometric recognition. ${ }^{1}$

Among all biometric techniques, the fingerprint recognition is the most popular method. This method has the following advantages: (1) universality-the population that has legible fingerprints exceeds the population that possesses the passports; (2) high distinctiveness-even identical twins who share the same DNA have different fingerprints; and (3) high performance - the fingerprint is one of the most accurate biometric characteristics with low FAR (false accept rate) and FRR (false reject rate). Already at the age of seven months, a fetus's fingerprints are

The authors are with the Biomedical Optics Laboratory, Biomedical Engineering Program, University of Houston, N207 Engineering Building 1, Houston, Texas 77204-4006. K. V. Larin's e-mail address is klarin@uh.edu.

Received 11 May 2006; revised 17 August 2006; accepted 23 August 2006; posted 29 August 2006 (Doc. ID 70746).

$0003-6935 / 06 / 369238-08 \$ 15.00 / 0$

(C) 2006 Optical Society of America fully developed, and characteristics of the fingerprints do not change throughout the lifetime except for injury or skin disease. However, after a small injury to a fingertip, the pattern will grow back as the fingertip heals. ${ }^{2}$ The uniqueness of the fingerprints can be determined by the pattern of minutia locations, ${ }^{3,4}$ local ridge orientation data, and ridgeorientation data combined with minutia locations. ${ }^{5}$ Therefore fingerprint recognition has become synonymous with the reliable method of personal identification. The FBI currently maintains more than 200 million fingerprint records on file. However, artificial finger dummies with embedded fingerprints, made using only $\$ 10$ worth of household supplies, may easily spoof common fingerprint systems. ${ }^{6}$ Therefore fingerprint recognition systems need to be improved to protect against different fraudulent methods.

During the past several years, significant improvements have been made by several scientific groups to enhance the robustness of the fingerprint readers based on the recognition of the surface topology. A smart card holder authentication system, which joined fingerprint verification with personal identification number (PIN) verification by applying a double random phase encoding scheme, was described in Ref. 7. By using an optimized template for core detection, the FRR was improved. Making use of a fast fingerprint enhancement algorithm, which could adaptively improve the clarity of ridge and valley structures of the input fingerprint images (based on the estimated local ridge orientation and frequency), a goodness index, and verification accuracy could also 


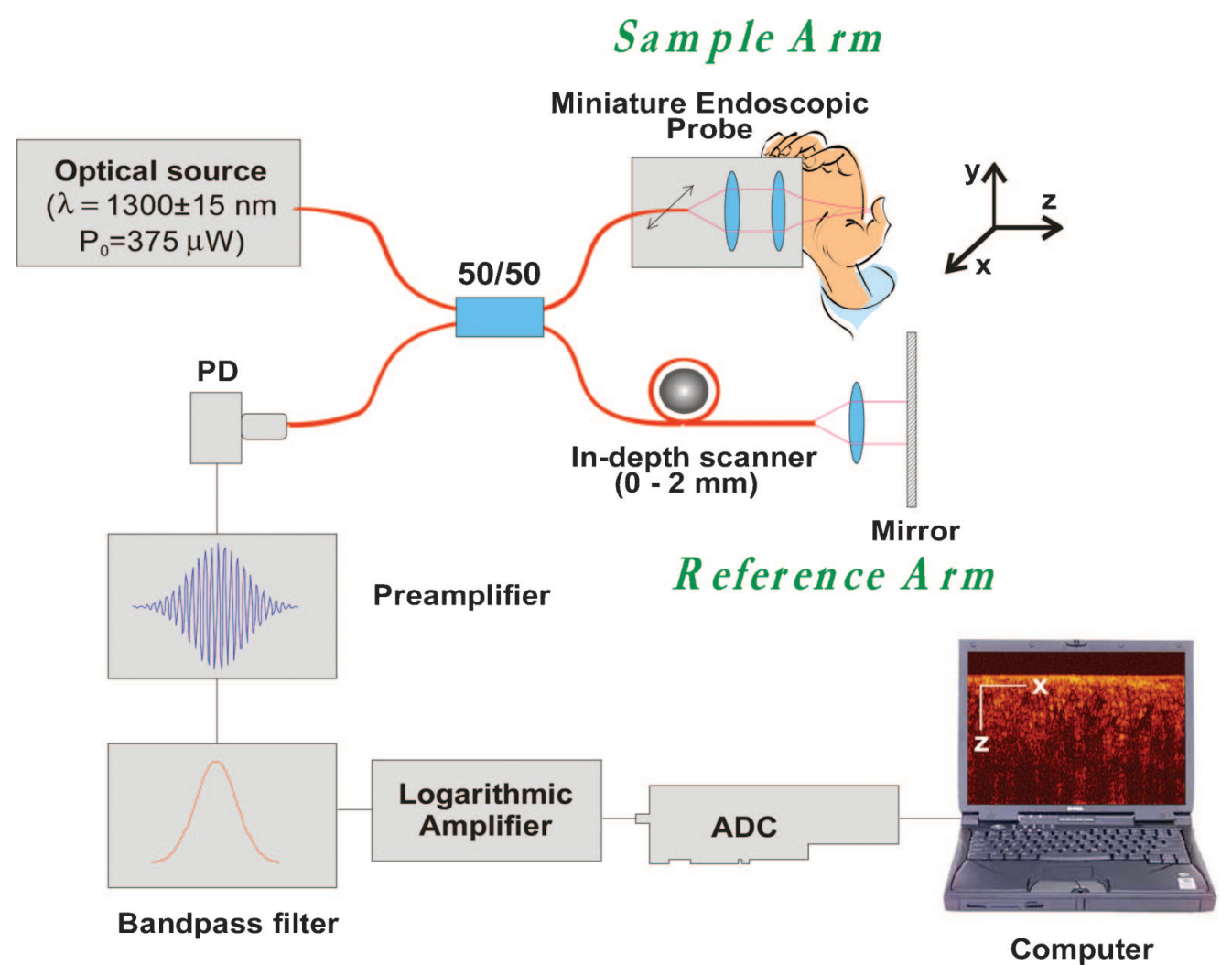

Fig. 1. (Color online) Schematic of the OCT system used in these studies. PD, photodetector; ADC, analog-to-digital converter.

be improved. ${ }^{8}$ However, these improvements in the fingerprint recognition methods concentrated on decreasing FRR and FAR and shortening scanning time. These improvements do not prevent system bypass by using artificial fingerprints. In this paper, we present the results on the application of the optical coherence tomography (OCT) technique, enhanced with an autocorrelation analysis, for identifying artificial materials commonly used for spoofing optical fingerprint scanning systems.

Since the introduction of the interferometric lowcoherence methods in the late $1980 \mathrm{~s},{ }^{9-11}$ the OCT technique has been widely applied in different fields, such as medical imaging diagnostics and material sciences. A typical time-domain OCT system is based on the Michelson interferometer configuration with a low coherent laser in a source arm, a moving mirror in a reference arm, an object under study in a sample arm, and a photodetector to measure the interference signal in a detection arm. In-depth scanning of the samples is produced by adjusting the lateral position of the mirror in the reference arm. Lateral scanning is realized through a second scanning mirror in the sample arm of the interferometer. The OCT technique has the unique ability of noninvasive in-depth and lateral scanning to capture $2 \mathrm{D}$ and $3 \mathrm{D}$ images with resolutions up to a few micrometers. These features could be used for the simple identification of additional artificial layers placed above real fingers by analyzing the OCT images. Furthermore, differences in optical properties between artificial materi- als and the real skin can be employed in automatic recognition systems based on, for instance, an autocorrelation analysis. With these unique capabilities, the artificial materials can be potentially recognized in a new generation of OCT-enhanced fingerprint systems.

\section{Materials and Methods}

\section{A. Experimental Setup}

Figure 1 shows a schematic of a time-domain OCT system used in these studies (Imalux Corp., Cleveland, Ohio). A low-coherent superluminescent laser diode with a wavelength of $1300 \pm 15 \mathrm{~nm}$ and an output power of $375 \mu \mathrm{W}$ was used as the optical source in this system. Light in the sample arm of the interferometer was directed into tissues using a single-mode optical fiber and a specially designed miniature endoscopic probe. The endoscopic probe allowed for the lateral scanning of the sample surface in the lateral direction ( $X$ axis). Light scattered from the sample and light reflected from the reference arm mirror formed an interferogram, which was detected by a photodiode. In-depth scanning was produced electronically by piezoelectric modulation of the fiber length. Two-dimensional images were obtained by scanning over the sample surface in the lateral direction ( $X$ axis) and in-depth ( $Z$ axis) scanning by the interferometer. The acquired images were $450 \times$ 450 pixels. In-depth scanning was up to $2.2 \mathrm{~mm}$ (in air), while lateral scanning was $2.4 \mathrm{~mm}$. The full 


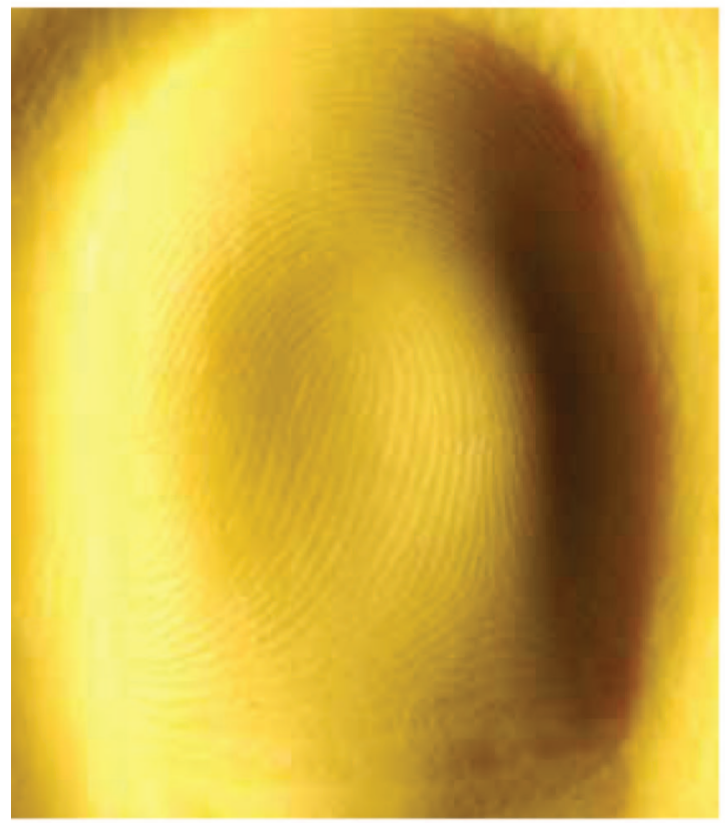

(a)

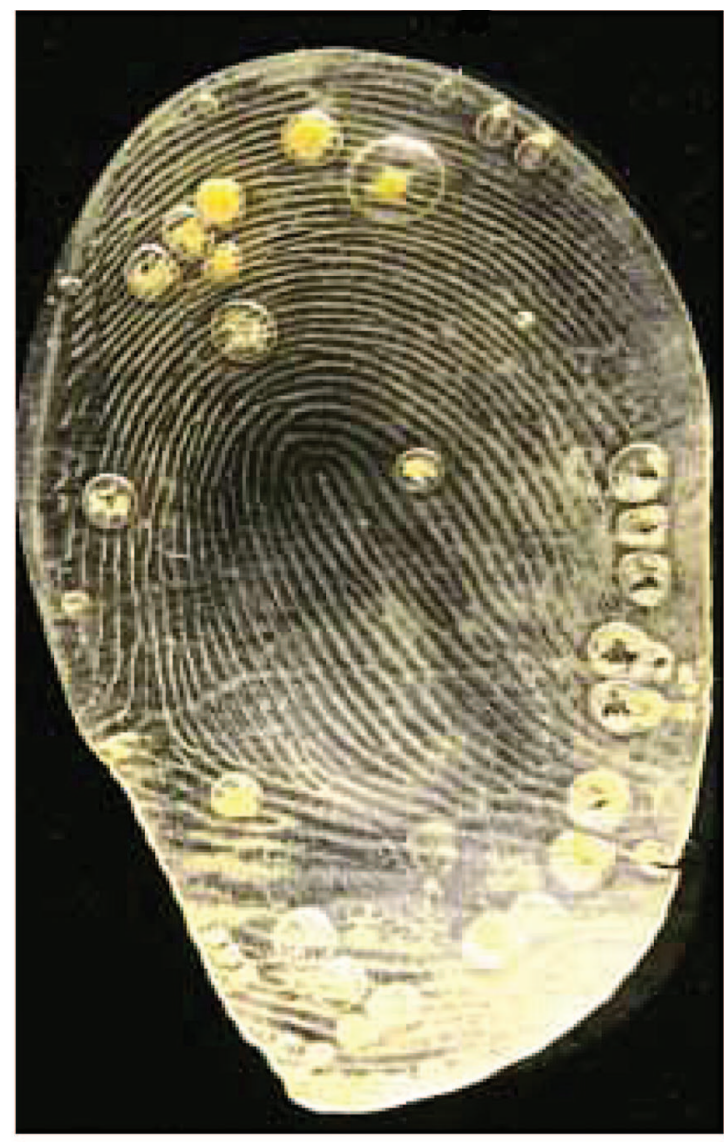

(b)

Fig. 2. (Color online) Gross pictures of (a) plasticene female mold and (b) artificial fingerprint dummy (male mold).

image acquisition rate was approximately $3 \mathrm{~s}$. The operation of the OCT system was fully controlled by a computer. The $2 \mathrm{D}$ images were averaged in a lateral direction (over $\approx 1 \mathrm{~mm}$, that was sufficient for speckle-noise suppression) into a single curve to obtain an OCT signal that represents a 1D distribution of light in depth (plotted in the logarithmic scale). The estimated system's resolution was $\sim 25$ $\mu \mathrm{m}$ in air.

\section{B. Materials}

A plasticene (Dixon Ticonderoga Company, Mexico), household cement (ITW Devcon Corp., Mass.), and a liquid silicon rubber (GE Silicones, General Electric Co., New York) were used to make artificial fingerprint dummies. We used general household materials that could be found in any supermarket and grocery store. The following procedures were followed to make an artificial fingerprint dummy (male mold) from the plasticene (female mold).

The plasticene was cut and kneaded into thick pieces for the preparation of a female mold. For the best imprinting of original fingerprint patterns, a finger was carefully washed with soap to get rid of the dust and tissue oil. Then, the finger was pressed firmly into the plasticene to leave the fingerprint pattern [female mold, Fig. 2(a)]. To prepare the male mold, we poured glue or liquid silicon rubber into the female mold. After natural solidification, the dummy was removed, and its fingerprint surface was carefully wiped to get rid of the plasticene pieces (internal impurities such as air bubbles or tiny pieces of plasticene were present, however, the OCT images were obtained from regions free from the structural defects). Hence the artificial fingerprint dummy (male mold) was ready for the experiments [Fig. 2(b)].

\section{Autocorrelation Analysis}

Autocorrelation analysis is a commonly used method in signal processing to analyze functions and series. It is a cross correlation of a signal with itself. The autocorrelation analysis is a useful technique for searching for repeating patterns, such as determining the presence of a periodic signal, which has been buried under the noise, e.g., speckle noise. ${ }^{12}$ Speckles are the spatially random coherent superposition (mutual interference) of light scattered from random scattering centers. In the OCT imaging of the scattering media, the speckle noise results from the coherent nature of laser radiation and the interferometric detection of the scattered light. ${ }^{13}$ The speckle noise substantially deteriorates resolution and the accuracy of the OCT images and, thus, several methods have been proposed to reduce its effect. ${ }^{13-18}$ However, the speckle noise bears useful information about tissue optical properties and could be utilized in tissue classification and monitoring 


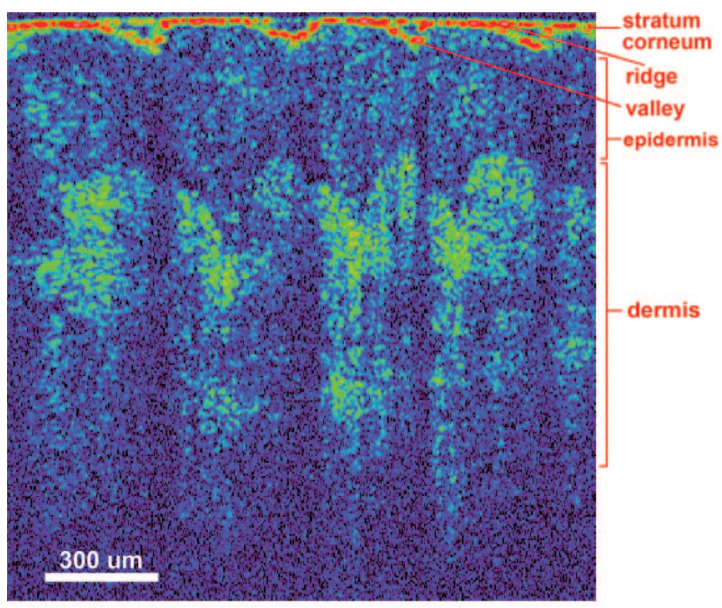

(a)

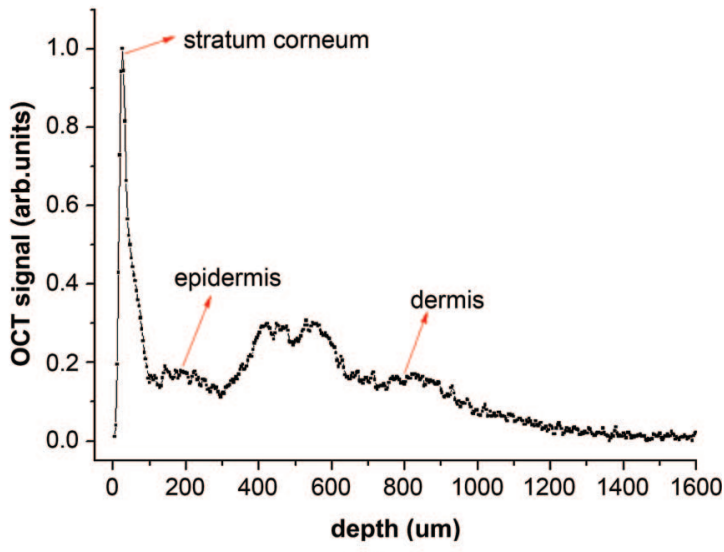

(b)

Fig. 3. (Color online) (a) Two-dimensional OCT images and (b) corresponding 1D OCT signal of a finger skin.

of different processes. ${ }^{19-22}$ Here, we applied an autocorrelation analysis to test whether the OCT images of artificial materials and real tissue can be distinguished.

Two-dimensional OCT images were converted into relative intensity values and then recorded in a square matrix $(450 \times 450$ pixels). Each column in the matrix contained information about one independent $Z$ scan of the OCT system. The discrete autocorrelation method was applied to process data in all columns. We defined the function $u(d)$, for a certain column intensity data in the image matrix, where $d$ was the depth with the range from 1 to 450 (corresponding to $0-1.6 \mathrm{~mm}$ depths in the sample with a refractive index of 1.4). Before autocorrelation analysis, we removed the mean value of $u(d)$ as $x(d)=$ $u(d)-\mu$, where $\mu=(1 / N) \sum_{n=1}^{N} u(d)$ was the mean value of the function $u(d)$. The discrete autocorrelation function for $x(d)$ was

$R_{x x}(r \Delta d)=\frac{1}{N-r} \sum_{n=1}^{N-r} x_{n} x_{n+1}=\frac{1}{N-r} \sum_{n=1}^{N-r} x(d) x(d+r \Delta d)$

in which $r=0,1,2, \ldots, m(m<N)$, and $r$ was the depth number, $\Delta d=1$ is the space interval unit (with the value of 1 OCT pixel), $N$ is the total available depth for a certain part (either the artificial material region or the real skin region) of an OCT signal curve, which was used in the autocorrelation analysis. The value $m$ in the autocorrelation analysis meant the maximum depth of the autocorrelation. Since we applied the autocorrelation function at the artificial material region and the human real finger region, respectively, the $N$ was different for them (we used $m=N-1$ in our program). For the artificial material region (the artificial fingerprint region), we used $m=50$, and for the real finger region, we used $m=100$.

With this algorithm, we calculated the autocorrelation function in each column of the OCT signals matrix and then averaged to find the algebra mean value. This algorithm was programmed in MATHEMATICA 5.0.

\section{Commercial Fingerprint Reader}

A commercially available fingerprint reader device (Microsoft Fingerprint Reader, model 1033, Redmond, Washington) was tested in our experiments. The fingerprint patterns of a volunteer's thumbs, forefingers, middle fingers, and ring fingers from both left and right hands were recorded and registered using a computer. The same fingers were used to prepare the artificial dummies. The dummies were placed on another person's finger and were scanned using both the fingerprint reader and the OCT system. Each dummy was tested 10-20 times with both systems and corresponding FARs were calculated.

\section{Results}

Figure 3(a) shows the typical OCT image of a finger skin. Three layers of human skin (stratum corneum, epidermis, and dermis) are clearly visible. The stratum corneum, which is a highly scattering tissue, is presented as the first thin layer in the image. The thickness of this layer is $\sim 15 \mu \mathrm{m}$. The ridges and valleys that determine fingerprint surface patterns can also be seen. The corresponding 1D OCT signal is shown in Fig. 3(b). Structural characteristic skin layers of the skin are also clearly visible.

Several materials, such as gelatin, silicon, wax, and agar, which could be used to make artificial fingerprints have been studied. Figure 4 illustrates a typical OCT image and its signal curve of a gelatin layer (25\% concentration) placed over a finger. The artificial gelatin layer and the human's skin layers beneath could be clearly detected from both Figs. 4(a) and 4(b). The gelatin layer is a homogeneous media, as illustrated by the OCT signal curve, and has a significantly lower scattering profile than that of the skin. The average thickness of the artificial layer was approximately $0.2 \mathrm{~mm}$. The characteristic layers of the human skin [as in Fig. 3(b)] can be clearly seen under the gelatin region. 


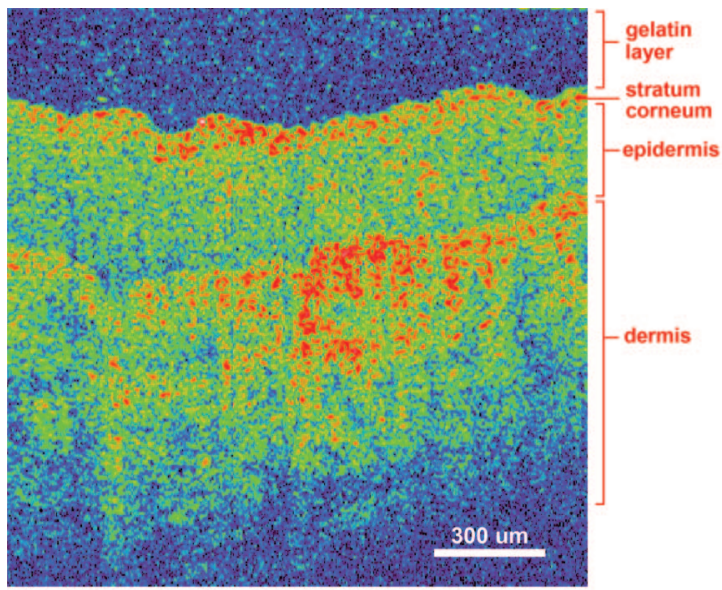

(a)

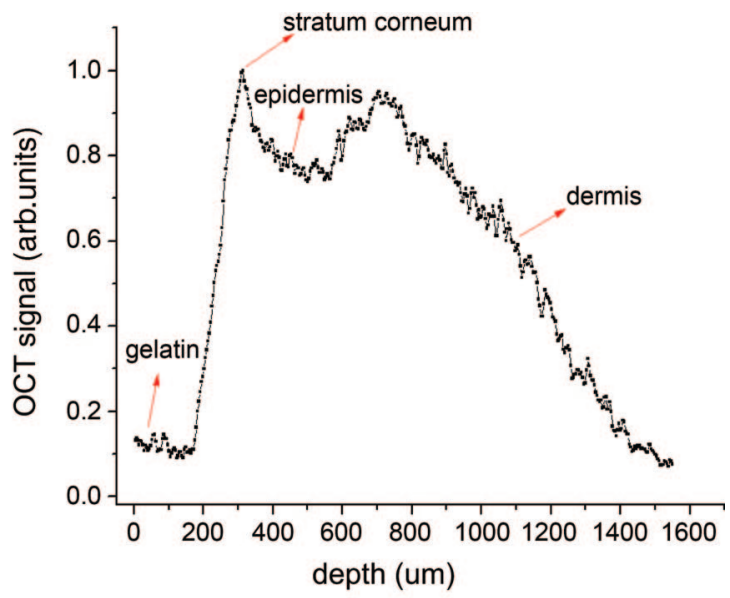

(b)

Fig. 4. (Color online) (a) OCT images of $25 \%$ gelatin with an average thickness of $0.2 \mathrm{~mm}$, (b) corresponding OCT signal.

Figure 5 shows the results obtained from the autocorrelation analysis of gelatin, agar, and real finger samples. The autocorrelation analysis was applied in the regions of OCT images corresponding to the artificial materials and the human skin. Depth, shown in pixels (1 pixel is approximately equal to $3.5 \mu \mathrm{m}$ ), was the interval where the OCT signal data were compared with itself. The autocorrelation function values for gelatin and agar fell sharply to zero with the depth increase due to the homogeneous structure of the artificial materials. Since the fluctuations of the speckle intensity in the OCT images were random and homogeneous in these regions, the autocorrelation function values at each depth were approximately zero. On the other hand, unlike the artificial material, the human skin is highly inhomogeneous tissue. When the autocorrelation analysis was applied to the skin, the generated autocorrelation function curves [Figs. 5(c) and 5(d)] nearly monotonically decreased with the depth increase and thus were

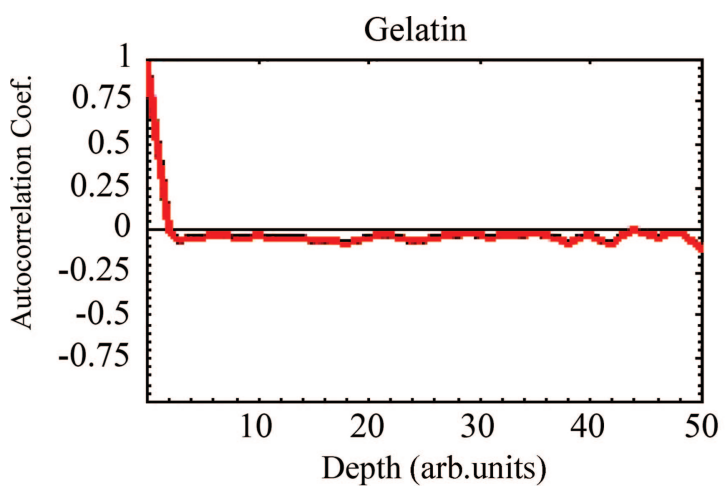

(a)

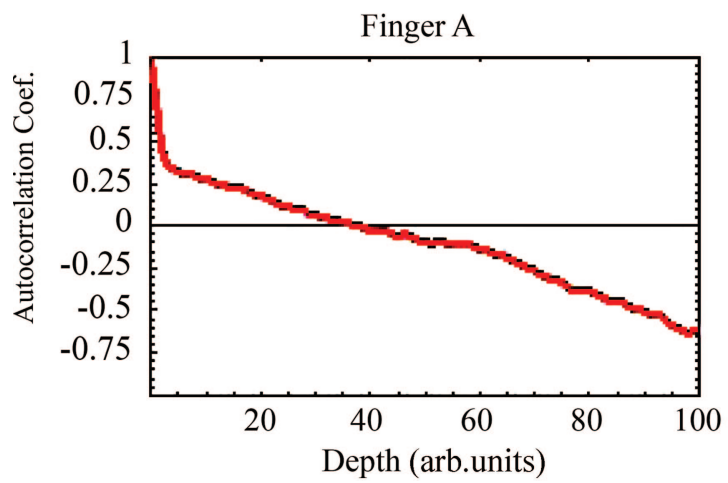

(c)

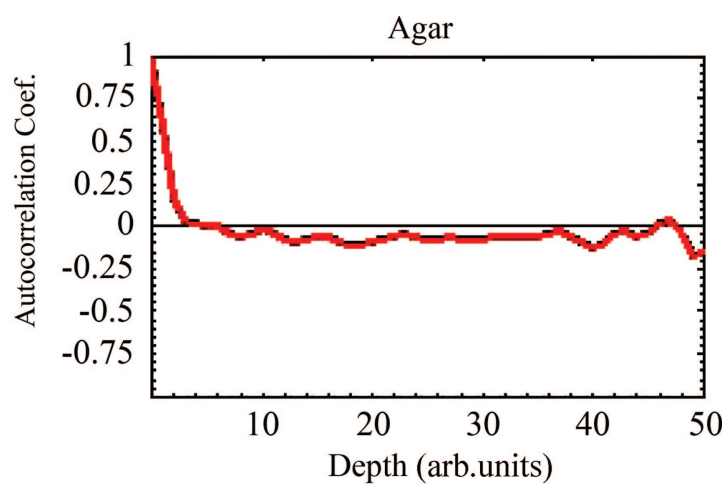

(b)

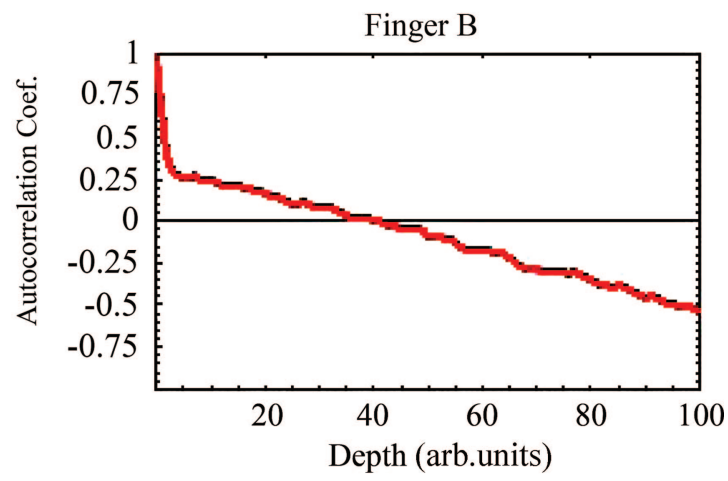

(d)

Fig. 5. (Color online) Autocorrelation curves for artificial materials of (a) gelatin and (b) agar and (c), (d) human finger, respectively. 


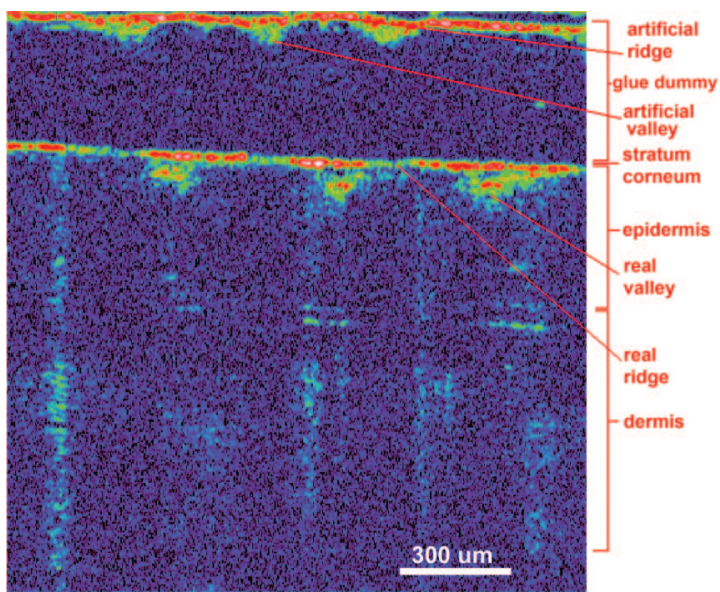

(a)

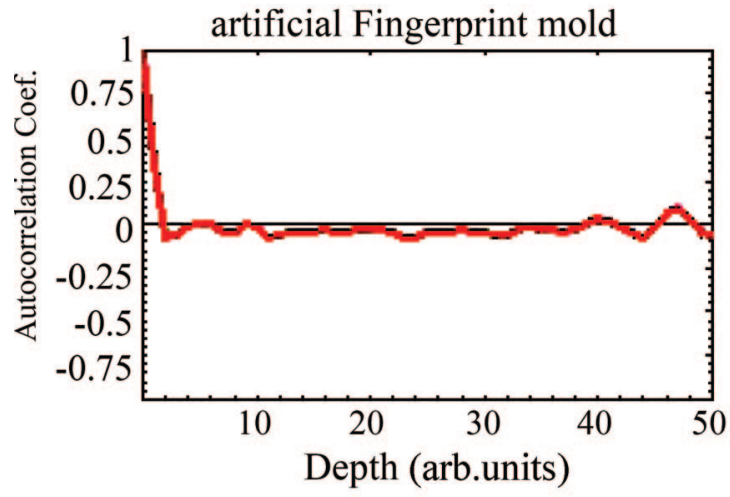

(c)

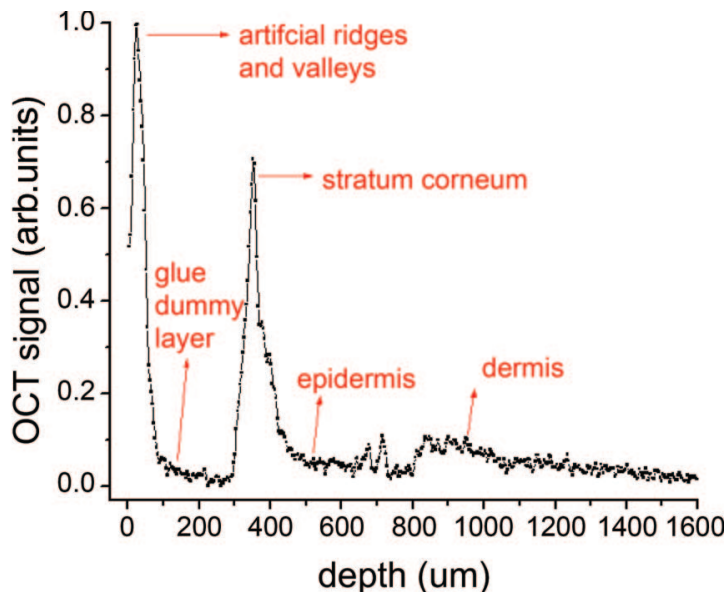

(b)

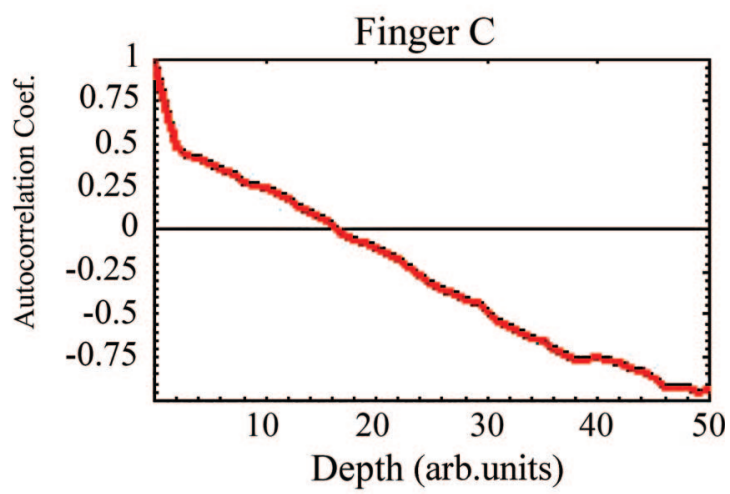

(d)

Fig. 6. (Color online) OCT images (a) obtained from the artificial fingerprint dummy over a real finger used to bypass the fingerprint reader device and (b) corresponding OCT signal curve. Autocorrelation curves were generated from the OCT image at the regions of (c) the artificial material and (d) human skin, respectively.

significantly different from those of the artificial materials. Therefore the autocorrelation analysis could potentially be used as a criterion for automatic and semiautomatic recognition of the artificial materials and the real fingers.

The artificial fingerprint dummies, similar to those shown in Fig. 2, were tested with the Microsoft fingerprint reader device and the OCT system. When these artificial dummies were applied to the reader, the FARs were from $80 \%$ to $100 \%$ (each mold was tried at least ten times). However, the artificial fingerprint dummies were always detected by the OCT system both visually (in 2D images and in corresponding OCT signal curves) and after processing with the autocorrelation analysis (Fig. 6). The number of trials per finger with the OCT system was at least 20 .

\section{Discussion}

Obtained results shown in Figs. 3-6 demonstrate the capability of the OCT technique for detecting artificial fingerprint dummies placed over real fingers. Most of the current fingerprint reading devices, such as the Microsoft fingerprint reader used in this study, are based on the surface analysis of fingerprint pat- terns (with low FAR and FRR). However, if an artificial fingerprint dummy was applied, these scanners might be easily spoofed. Since 1990, several fingerprint sensors have been tested using dummy fingers. All tested sensors accepted the dummy as a real finger, almost from the first attempt. ${ }^{23}$ Since OCT could provide high-resolution in-depth information about tissue structure, the additional layers, which are not characteristic for the normal skin, could be easily identified (Figs. 4 and 6). In this study, the artificial dummies were distinguished at all times using the OCT technique.

Previously, we demonstrated that optical properties (e.g., scattering coefficients) as well as physical properties (e.g., thicknesses) of artificial layers and characteristic layers of human skin might be close to each other. ${ }^{24}$ In this paper, we exploited the differences in the homogeneity between these objects (unlike the skin, the scattering particles in artificial materials were homogeneously distributed through the depth). Moreover, the sizes of the scattering particles might be quite different. Therefore the size and distribution of the speckles obtained from these structures would be different. Here, we applied autocorrelation space analysis to the regions of the OCT 


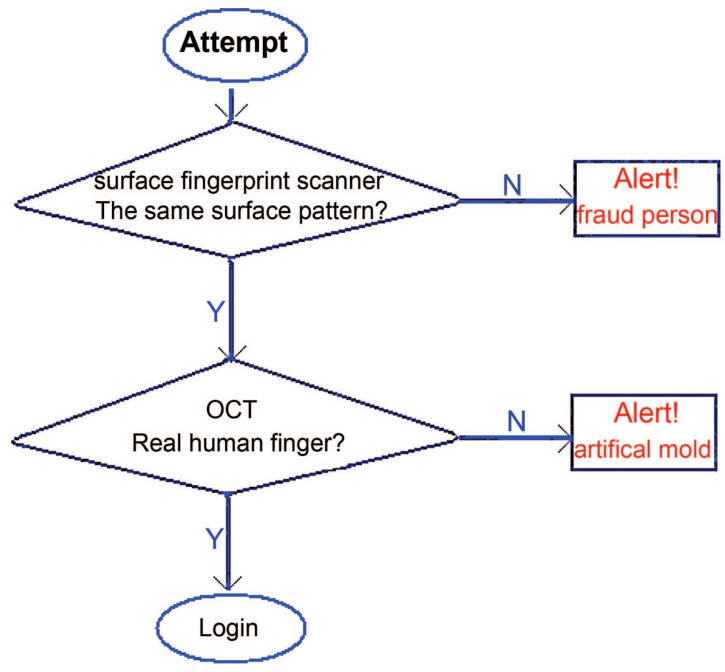

(a)

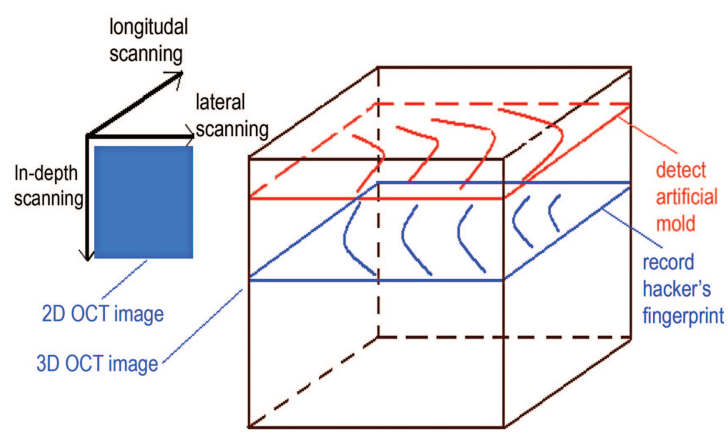

(b)

Fig. 7. (Color online) (a) Flow chart for an OCT-enhanced surface fingerprint scanner; (b) sketch for the reconstruction of the OCT 3D image from a false fingerprint.

images corresponding to the artificial material and real skin. We found that the autocorrelation function curves clearly indicated the presence of the artificial materials on the human finger. The autocorrelation analysis gave a mathematical criterion to distinguish the skin from the artificial materials. Therefore the autocorrelation analysis might be used as one of the effective tools in an automatic fingerprint recognition system, enhanced with OCT [Fig. 7(a)].

In addition, an OCT system with 3D image acquisition could be utilized as a fingerprint identification system by itself. As shown in Figs. 3 and 6, OCT images can visualize not only tissue layers but also surface ridges and valleys that constitute the fingerprint pattern. Therefore 3D images of human skin can provide information about fingerprint patterns as well. Moreover, Fig. 6 also demonstrates that the fingerprint patterns could simultaneously be detected from a dummy placed on a finger and the finger skin itself. Hence it might be possible to record and analyze fingerprint patterns from the artificial dummy as well as from the real skin simultaneously, as shown schematically in Fig. 7(b).

\section{Conclusion}

In this study, we demonstrated that the OCT technique could be successfully applied for the identification of artificial materials commonly used to make fake fingerprints. While the commercially available fingerprint reader system was easily bypassed using fingerprint dummies, the artificial materials were detected and recognized using the OCT system at all times. To summarize, our results demonstrated that (1) current fingerprint systems based on surface scanning could be easily spoofed by a fingerprint dummy; (2) high-resolution OCT 2D images and corresponding signal curves revealed the presence of artificial materials at all times; (3) autocorrelation analysis could potentially be used in automatic fingerprint recognition systems; (4) a fingerprint recognition system, combined with OCT, could be more secure and integral. Our future studies will be focused on OCT $3 \mathrm{D}$ image acquisition and reconstruction algorithms for biometric identification based on the fingerprints.

This work was supported by a grant from the University of Houston (ST 35906). We thank Mohamad M. Ghosn, Ravi Kiran Manapuram, and Stephen Pinchback for their help with experiments, discussion of the data, and the photographs of the fingerprints.

\section{References}

1. S. Prabhakar, S. Pankanti, and A. K. Jain, "Biometric recognition: security and privacy concerns," IEEE Security Privacy 1, 33-42 (2003).

2. D. Maltoni, D. Maio, A. K. Jain, and S. Prabhakar, Handbook of Fingerprint Recognition (Springer, 2003).

3. J. H. Chang and K. C. Fan, "Fingerprint ridge allocation in direct gray-scale domain," Pattern Recogn. 34, 1907-1925 (2001).

4. E. Nikodemusz-Szekely and V. Szekely, "Image recognition problems of fingerprint identification," Microprocess. Microsys. 17, 215-218 (1993).

5. P. R. Vizcaya and L. A. Gerhardt, "Nonlinear orientation model for global description of fingerprints," Pattern Recogn. 29, 1221-1232 (1996).

6. T. Matsumoto, H. Matsumoto, K. Yamada, and S. Hoshino, "Impact of artificial "gummy" fingers on fingerprint systems," in Proc. SPIE 4677, 275-289 (2002).

7. H. Suzuki, M. Yamaguchi, M. Yachida, N. Ohyama, H. Tashima, and T. Obi, "Experimental evaluation of fingerprint verification system based on double random phase encoding," Opt. Express 14, 1755-1766 (2006).

8. H. Lin, W. Yifei, and A. Jain, "Fingerprint image enhancement: algorithm and performance evaluation," IEEE Trans. Pattern Anal. Mach. Intell. 20, 777-789 (1998).

9. J. G. Fujimoto, S. De Silvestri, E. P. Ippen, C. A. Puliafito, R Margolis, and A. Oseroff, "Femtosecond optical ranging in biological systems," Opt. Lett. 11, 150-153 (1986).

10. A. F. Fercher, K. Mengedoht, and W. Werner, "Eye-length 
measurement by interferometry with partially coherent light," Opt. Lett. 13, 186-188 (1988).

11. D. Huang, E. A. Swanson, C. P. Lin, J. S. Schuman, W. G. Stinson, W. Chang, M. R. Hee, T. Flotte, K. Gregory, C. A. Puliafito, and J. G. Fujimoto, "Optical coherence tomography," Science 254, 1178-1181 (1991).

12. R. N. Bracewell, The Fourier Transform and Its Applications, 3rd ed., McGraw-Hill Series in Electrical and Computer Engineering. Circuits and Systems (McGraw-Hill, 2000), pp. xx, 616.

13. J. M. Schmitt, S. H. Xiang, and K. M. Yung, "Speckle in optical coherence tomography,” J. Biomed. Opt. 4, 95-105 (1999)

14. J. Rogowska and M. E. Brezinski, "Evaluation of the adaptive speckle suppression filter for coronary optical coherence tomography imaging," IEEE Trans. Med. Imaging 19, 12611266 (2000).

15. N. Iftimia, B. E. Bouma, and G. J. Tearney, "Speckle reduction in optical coherence tomography by path length encoded angular compounding,” J. Biomed. Opt. 8, 260-263 (2003).

16. A. I. Kholodnykh, I. Y. Petrova, K. V. Larin, M. Motamedi, and R. O. Esenaliev, "Precision of measurement of tissue optical properties with optical coherence tomography," Appl. Opt. 42, 3027-3037 (2003).

17. M. Pircher, E. Gotzinger, R. Leitgeb, A. F. Fercher, and C. K. Hitzenberger, "Speckle reduction in optical coherence tomog- raphy by frequency compounding," J. Biomed. Opt. 8, 565-569 (2003).

18. J. Kim, D. T. Miller, E. Kim, S. Oh, J. Oh, and T. E. Milner, "Optical coherence tomography speckle reduction by a partially spatially coherent source," J. Biomed. Opt. 10, 064034 (2005).

19. D. A. Zimnyakov, V. P. Ryabukho, and K. V. Larin, "Microlens effect due to the diffraction of focused beams on large-scale phase screens," JETP Lett. 20, 14-19 (1994).

20. K. W. Gossage, T. S. Tkaczyk, J. J. Rodriguez, and J. K. Barton, "Texture, analysis of optical coherence tomography images: feasibility for tissue classification,” J. Biomed. Opt. 8, $570-575$ (2003).

21. D. A. Zimnyakov and M. A. Vilensky, "Blink speckle spectroscopy of scattering media," Opt. Lett. 31, 429-431 (2006).

22. D. A. Zimnyakov, D. N. Agafonov, A. P. Sviridov, A. I. Omel'chenko, L. V. Kuznetsova, and V. N. Bagratashvili, "Speckle-contrast monitoring of tissue thermal modification," Appl. Opt. 41, 5989-5996 (2002).

23. T. van der Putte and J. Keuning, "Biometrical fingerprint recognition: don't get your fingers burned," presented at the Fourth Working Conference on Smart Card Research and Advanced Applications, Bristol, UK, 20-22 September 2000.

24. R. K. Manapuram, M. Ghosn, and K. V. Larin, "Identification of artificial fingerprints using optical coherence tomography technique," Asian J. Phys. 15, 15-27 (2006). 